Arq. Bras. Med. Vet. Zootec., v.64, n.6, p.1442-1448, 2012

\title{
Concentração de lactato e glicemia em cadelas e neonatos nascidos de cesariana
}

[Lactato concentration in bitch and canine neonates born through cesarean section]

L.M.C. Bueno, M.D. Lopes, M.L.G. Lourenço, N.C. Prestes, R.K. Takahira, A.A.P. Derussi, M.J. Sudano

Faculdade de Medicina Veterinária e Zootecnia - UNESP - Botucatu, SP

\section{RESUMO}

Avaliaram-se a concentração de lactato e a glicemia em sete cadelas em trabalho de parto submetidas à cesariana e em seus respectivos neonatos. O lactato materno foi mensurado utilizando-se medidor portátil e pelo método enzimático colorimétrico. Nos neonatos avaliou-se também o peso ao nascimento. Os valores de lactato materno e neonatal foram estatisticamente diferentes, sendo mais elevado nos neonatos. Ao nascimento, as cadelas e os neonatos encontravam-se normoglicêmicos, e não se observou correlação entre a glicemia de ambos. Os valores de lactato obtidos pelo o analisador portátil $(3,5 \pm 0,6 \mathrm{mmol} / \mathrm{L})$ foram mais altos do que os obtidos pelo método enzimático colorimétrico $(1,6 \pm 0,6 \mathrm{mmol} / \mathrm{L})$. Observou-se correlação significativa entre o peso do neonato e a concentração de lactato, isto é, os filhotes de menor peso apresentaram maior concentração de lactato.

Palavras-chave: cadela, neonato, lactato, glicemia, accutrend

\begin{abstract}
This work evaluated the concentration of lactate and blood glucose in seven bitches in labor subject to caesarean section and in their newborns. The lactate concentration was measured using a portable analyzer and enzymatic colorimetric method. In neonates, the birth weight was evaluated. The maternal and neonatal lactate values were statistically different, being higher in newborns. At birth, the bitches and the newborns had normal glucose status, and no correlation was observed between blood glucose in either. The lactate concentration obtained by the portable analyzer $(3.5 \pm 0.6 \mathrm{mmol} / \mathrm{L})$ was higher than that obtained with the enzymatic colorimetric method $(1.6 \pm 0.6 \mathrm{mmol} / \mathrm{L})$. There was significant correlation between the neonate weight and lactate concentration and in puppies with lighter weight there was a higher concentration of lactate.
\end{abstract}

Keywords: bitch, neonate, lactate, glucose, accutrend

\section{INTRODUÇÃO}

A mensuração da concentração de lactato é uma técnica minimamente invasiva que pode ser realizada com amostras de sangue venoso (McMichael et al., 2005), sendo que em humanos tem se mostrado uma ferramenta útil para diagnóstico, monitoração e prognóstico de uma ampla gama de síndromes clínicas. Seu uso em pequenos animais vem sendo preconizado, e vários estudos foram feitos demonstrando seu papel na medicina intensiva (Pang e Boysen, 2007; Allen et al., 2008). Os valores de lactato

Recebido em 18 de julho de 2011

Aceito em 10 de setembro de 2012

E-mail: mege@uol.com.br descritos em cães adultos foram de $1,57 \pm 0,47 \mathrm{mmol} / \mathrm{L}$ (Hughes et al.,1999) ou de 1,1 a 2,5mmol/L (Lagutchik et al., 1998).

O uso da concentração de lactato sanguíneo como indicador da gravidade do prognóstico e da doença constitui-se uma prática comum na medicina veterinária, especialmente com a validação de analisadores portáteis (Evans, 1987; Ferasin, et al., 2007; Stevenson et al., 2007; Castagnetti, 2010). No entanto, poucos autores descreveram a concentração de lactato de neonatos caninos em estado crítico, e não há muitos estudos publicados sobre o uso de analisadores portáteis em cães neonatos. 
Segundo Koliski et al. (2005), em estudo sobre a utilização de lactato sérico como marcador prognóstico em crianças gravemente doentes, a maioria dos pacientes com concentração de lactato acima de $2 \mathrm{mmol} / \mathrm{L}$ evidenciou sinais clínicos de hipoperfusão. A normalização ou diminuição da concentração, a partir de 24 horas de internação, esteve significativamente relacionada com a maior probabilidade de sobrevivência (American ..., 1986; Barroso et al., 2006).

Em estudo comparativo em cães adultos e filhotes, a partir de quatro, 10, 16, 28, 70 e 80 dias de idade, a concentração de lactato em filhotes foi mais elevada nos neonatos com quatro, 10,16 e 28 dias de idade quando comparada à de cães adultos $(1,80 \pm 0,84 \mathrm{mmol} / \mathrm{L})$. $\mathrm{O}$ intervalo de referência para a concentração de lactato para neonatos com quatro dias de idade foi de 1,07-6,59mmol/L, e para os filhotes com 10-28 dias de idade de 0,80-4,60mmol/L (McMichael et al., 2005).

De acordo com Groppetti et al. (2010), o reconhecimento da acidose fetal por meio da avaliação de lactato umbilical juntamente com a classificação do escore Apgar, e o monitoramento da atividade uterina durante o parto, podem representar um sistema avançado na avaliação do neonato canino. A mensuração da concentração de lactato umbilical e sua correlação com a morbidade e a mortalidade em 48 horas após o nascimento provou ser útil para prevenção da mortalidade neonatal canina. A concentração de lactato umbilical de $5 \mathrm{mmol} / \mathrm{L}$ foi estabelecida como valor de referência para distinção entre filhotes saudáveis e em angústia neonatal. Valores mais elevados relacionavam-se com filhotes em sofrimento, enquanto os valores mais baixos caracterizavam filhotes vigorosos. Concentrações de lactato inferiores a $5 \mathrm{mmol} / \mathrm{L} \mathrm{e}$ índice de Apgar superior a 9, analisados conjuntamente com a duração do parto, foram considerados bons índices prognósticos na neonatologia canina (Groppetti et al., 2010).

Vivan et al. (2009) também demonstraram a validade do lactato como indicador de sofrimento em cães neonatos durante o nascimento. O lactato no sangue venoso de neonatos oriundos de parto normal foi avaliado ao nascimento, aos quatro e aos 30 dias de idade. Ao nascimento $(6,26 \pm 2,7 \mathrm{mmol} / \mathrm{L})$, o lactato foi significativamente mais elevado quando comparado ao quarto $(2,37 \pm 0,99 \mathrm{mmol} / \mathrm{L})$ e ao $30^{\circ}$ dia de idade $(2,57 \pm 1,09 \mathrm{mmol} / \mathrm{L})$. Foram observados índices elevados de lactato nos neonatos que vieram a óbito $(13,1 \pm 0,9 \mathrm{mmol} / \mathrm{L})$ quando comparados aos que sobreviveram $(5,3 \pm 1,9 \mathrm{mmol} / \mathrm{L})$.

A correlação do lactato sérico com a vitalidade de neonatos de cães nascidos de cesariana sob anestesia geral inalatória foi avaliada por Souza et al. (2006). Nesse estudo houve diferença entre o lactato dos filhotes e das mães e não houve correlação entre o lactato dos filhotes e o Apgar, contrariamente ao descrito por Groppetti et al. (2010); por outro lado, houve correlação negativa significativa entre os níveis de lactato dos filhotes e a média das frequências cardíacas fetais. Segundo os autores, os reflexos de vitalidade neonatal, como o de dor e de sucção, foram ausentes em $91,3 \%$ e $82,6 \%$ dos neonatos ao nascimento, permanecendo ausentes em $52,2 \%$ e 43,5\% 10 minutos após o nascimento, respectivamente.

Em cães neonatos, os níveis glicêmicos são menores imediatamente após o nascimento, sendo os valores de referência para cães com um a três dias de idade de 76 a $155 \mathrm{mg} / \mathrm{dL}$. Após o terceiro dia de vida, a glicemia se eleva no filhote em lactação, atingindo valores em torno de 101 a $161 \mathrm{mg} / \mathrm{dL}$, não ocorrendo mais variações significativas nos níveis glicêmicos do oitavo dia até o desmame. Ao nascimento, a menor concentração de glicose em neonatos deve-se à insuficiência dos mecanismos regulatórios da glicose sanguínea bem como pela imaturidade hepática do neonato (gliconeogênese hepática ineficaz, menores estoques de glicogênio hepático) e pela glicosúria, devido à imaturidade renal (Gorman, 2011).

A determinação da glicemia em fêmeas no periparto e em neonatos nascidos em eutocia, distocia corrigida por manobra obstétrica e/ou cesariana ou por indução medicamentosa (ocitocina e gluconato de cálcio) foi descrita (Lucio et al., 2008). As fêmeas encontravamse normoglicêmicas, independentemente do momento e do tipo do parto, embora os níveis glicêmicos do pré-parto fossem inferiores aos encontrados uma hora após o parto. Já em neonatos, embora normoglicêmicos ao 
nascimento, a glicemia foi superior em partos distócicos e por indução medicamentosa.

Os estudos avaliando a glicemia em cães nascidos por cesariana são escassos, contudo a acurada avaliação é fundamental no pós-parto imediato, particularmente nos neonatos oriundos de partos distócicos, mais propensos à hipoglicemia Lawer (2008).

Pelo exposto, o objetivo do presente trabalho foi avaliar a concentração de lactato e a glicemia em cadelas submetidas à cesariana e nos neonatos $\mathrm{e}$ comparar duas metodologias de análise de lactato pelo analisador portátil (Accutrend LactatoRoche) e pelo método enzimático colorimétrico.

\section{MATERIAIS E MÉTODOS}

Foram utilizadas sete cadelas adultas, de diferentes raças, gestantes em trabalho de parto, com idade entre um a 10 anos, em boas condições corporais. Após exame clinico geral e específico do aparelho genital, avaliação ultrassonográfica e constatação da viabilidade fetal, as parturientes com indicação de cesariana foram encaminhadas para a anestesia e procedimento cirúrgico.

Previamente ao procedimento anestésico, as cadelas foram submetidas a tricotomia na região da veia jugular, antissepsia e colheita de sangue. $\mathrm{O}$ sangue foi colhido preferencialmente sem garroteamento ou com um mínimo de estase venosa. Uma única gota de sangue total foi utilizada para a avaliação direta do lactato e da glicose sanguínea minutos após a coleta, por intermédio de analisadores portáteis (Accutrend Lactato-Roche; One Touch Glicemia Ultra ${ }^{\circledR}$ Johnson \& Johnson). No presente estudo, utilizaram-se os valores de referência para lactato em adultos segundo Stevenson et al. (2007), de 1,2-3,1 mmol/L e da glicose, segundo Kaneko (1997), de 70 a $110 \mathrm{mg} / \mathrm{dL}$.

Foram retirados, em média, um volume de $5,0 \mathrm{~mL}$ de sangue em seringas de $10,0 \mathrm{~mL}$ e agulhas 30x08. O sangue foi centrifugado por 10 minutos para obtenção de plasma. Esse material foi armazenado sob temperatura de congelamento $\left(-10^{\circ} \mathrm{C}\right)$. As amostras de plasma foram obtidas apenas com anticoagulantes contendo fluoreto, pois esse anticoagulante estabiliza o lactato sanguíneo ao inibir a glicólise. O lactato no plasma materno também foi dosado pelo método enzimático colorimétrico, utilizando-se os valores de referência para adultos segundo Hughes (1999; 2006), de 0,3-2,5mmol/L.

As fêmeas foram então submetidas ao procedimento anestésico com $0,05 \mathrm{mg} / \mathrm{kg}$ acepromazina e $0,5 \mathrm{~g} / \mathrm{kg}$ morfina, por via intramuscular como medicação pré-anestésica e após 10 minutos aplicação de $5 \mathrm{mg} / \mathrm{kg}$ ketamina e $0,25 \mathrm{mg} / \mathrm{kg}$ de diazepam por via intravenosa e manutenção da anestesia inalatória com halotano. Como terapia pós-operatória, foi recomendado o uso de $0,1 \mathrm{mg} / \mathrm{kg}$ de meloxican durante dois dias e antibioticoterapia utilizando penicilina benzatina $30.000 \mathrm{UI} / \mathrm{kg}$, por via intramuscular, a cada 48 horas.

Durante o procedimento cirúrgico, os fetos foram cuidadosamente retirados e separados da placenta, e, realizada a ligadura do cordão umbilical, submetidos à assistência neonatal limpeza dos envoltórios fetais, desobstrução das vias aéreas, secagem com compressa cirúrgica estéril - e em seguida procedeu-se à colheita de sangue da veia jugular para dosagem do lactato e da glicemia. Uma única gota de sangue total foi utilizada para a avaliação direta do lactato e da glicose, por intermédio de analisadores portáteis. No presente estudo, utilizaram-se os valores de referência de lactato em analisador portátil para neonatos segundo McMichael et al. (2005), de 1,07-6,59mmol/L, e para glicose, segundo Gorman (2011), de 76 a 155mg/dL. Os filhotes foram pesados e mantidos em local aquecido até a completa recuperação pós-operatória da parturiente.

A metodologia estatística utilizou a ANOVA após a confirmação da distribuição normal, usando o teste Kolmogorov-Smirnov $(\mathrm{P}<0,15)$. Para todas as análises foi adotada a significância de 5\%. As correlações de Pearson foram calculadas entre as variáveis maternas - lactato e glicemia -, dos neonatos - lactato, glicemia e peso - e maternas e dos neonatos - lactato, glicemia. Nesta última, utilizaram-se as médias dos filhotes de cada ninhada.

O presente estudo foi aprovado pelo Comitê de Ética no Uso de Animais (CEUA-FMVZ), sob o protocolo $55 / 2010$. 


\section{RESULTADOS E DISCUSSÃO}

Os valores de lactato das cadelas (Tab. 1) situaram-se discretamente acima das concentrações normais para a espécie, utilizandose medidores portáteis, cuja variação, segundo Stevenson et al. (2007) e Allen et al. (2008), é de $1,2-3,1 \mathrm{mmol} / \mathrm{L}$, e dentro das concentrações descritas por Hughes (1999; 2006), para o método enzimático, de $0,3-2,5 \mathrm{mmol} / \mathrm{L}$. A glicemia das cadelas também estava dentro dos padrões de referência propostos para a espécie por Kaneko (1997), de 70 a 110mg/dL.

Tabela 1. Média e erro-padrão das médias (E $\mathrm{Pm}$ ) da concentração de lactato (mmo/L) obtidas pelo método do analisador portátil e da concentração de glicose (g/dl) nas parturientes

\begin{tabular}{lc}
\hline \multicolumn{1}{c}{ Variável } & Média $\pm \mathrm{E} \mathrm{Pm}$ \\
\hline Lactato (mmol/L) & \\
Medidor portátil & $3,5 \pm 0,6$ \\
Método enzimático & $1,6 \pm 0,6$ \\
\hline Glicemia $(\mathrm{mg} / \mathrm{dL})$ & $94,3 \pm 6,4$ \\
\hline Lactato X Glicemia & $\mathrm{r}=0,38 ; \mathrm{P}=0,39$ \\
\hline
\end{tabular}

O lactato sanguíneo das cadelas submetidas à cesariana não demonstrou alteração significativa, resultado esse compatível com o citado por Souza et al. (2006), em que o lactato de cadelas previamente ao procedimento cirúrgico de cesariana foi de $2,49 \pm 0,87 \mathrm{mmol} / \mathrm{L}$. A concentração de lactato materno previamente ao parto não reflete a redução da perfusão tecidual uterina e consequentemente a fetal. Segundo Groppetti et al. (2010), o método ideal para identificação intra-parto de hipóxia fetal em humanos é a análise de lactato sanguíneo do feto, colhido do couro cabeludo ou do cordão umbilical.

Em relação à glicemia das cadelas em periparto, os resultados obtidos no presente estudo foram semelhantes aos citados por Lucio et al. (2008), em que ocorreu predomínio da normoglicemia em distocias corrigidas por manobras obstétricas ou por cesariana. A manutenção da concentração glicêmica em fêmeas em distocia e submetidas a cesariana deve-se provavelmente ao fato de haver a liberação de hormônio cortisol, que estimula a neoglicogênese hepática, promovendo a conversão de aminoácidos em glicose. Outros hormônios, como a adrenalina e insulina, envolvidos em situações de estresse, também influenciariam o status glicêmico, mantendo as concentrações adequadas de glicose.

Neste estudo, não se observou correlação entre concentração de lactato e glicemia materna $(\mathrm{P}=0,39)$.

Os valores de lactato nos neonatos (Tab. 2) situaram-se acima dos níveis descritos para neonatos com quatro dias de idade, utilizando-se medidores portáteis, cuja variação, segundo McMichael et al. (2005) e McMichael (2005), é de 1,07-6,59mmol/L. O maior valor de lactato encontrado nos neonatos do presente estudo foi de $18,2 \mathrm{mmol} / \mathrm{L}$ e o menor, 3,2 mmol/L. Os resultados também foram semelhantes aos descritos por Groppetti et al. (2010), que determinaram a concentração de lactato em neonatos nascidos de cesariana emergencial de $8.3 \pm 5.9 \mathrm{mmol} / \mathrm{L}$. Segundo esses autores, o valor de referência de lactato para distinção entre filhotes saudáveis e em angústia neonatal é de $5 \mathrm{mmol} / \mathrm{L}$, em sangue do cordão umbilical. Os valores foram mais elevados do que os encontrados por Vivan et al. (2009) e por Souza et al. (2006), no sangue venoso, cujos valores ao nascimento foram, respectivamente, de $6,26 \pm 2,7 \mathrm{mmol} / \mathrm{L}$ e de $5,56 \pm 2,31 \mathrm{mmol} / \mathrm{L}$.

Embora o lactato sanguíneo seja um marcador útil de perfusão tecidual inadequada, e sua elevação no feto e no neonato ocorra pelo metabolismo anaeróbico desencadeado pela hipóxia em diversas espécies, neste estudo a correlação entre o óbito e hiperlactatemia intensa, descrito por Vivan et al. (2009), em que filhotes com lactato acima de $13,1 \pm 1,9 \mathrm{mmol} / \mathrm{L}$ vieram a óbito, e por Groppetti et al. (2010), não foi observada. Aliás, todos os neonatos permaneceram vivos por 24 horas após o nascimento. Desse modo, a mensuração do lactato não deve se constituir na única forma de avaliação do grau de depressão em cães nascidos de cesariana.

A glicemia dos neonatos também se encontrava dentro dos padrões normais propostos por Gorman (2011), de 76 a 155mg/dL, e de acordo com o descrito por Lucio et al. (2008), que detectaram normoglicemia neonatal ao nascimento, com prevalência de valores mais elevados nos filhotes nascidos por cesariana em razão do maior estresse ao nascimento, e por Lawer (2008). A hiperglicemia nas primeiras 
horas de vida é causada pela liberação de catecolaminas e de cortisol; posteriormente pode haver hipoglicemia pela depleção dos estoques reduzidos de glicogênio hepático neonatal. Portanto, a terapia empírica com glicose oral ou intravenosa durante a reanimação neonatal deve ser realizada somente após a avaliação da glicemia.

Não se observou correlação entre lactato e glicemia neonatal $(\mathrm{P}=0,44)$ nem entre peso e glicemia $(P=0,82)$, contudo correlação negativa entre peso e lactato $(\mathrm{r}=-0,67 ; \mathrm{P}=0,0002)$ (Fig. 1) foi observada, isto é, os filhotes com menor peso apresentavam lactato mais elevado. A média de peso dos neonatos ao nascimento foi de $218,5 \pm 12,7$ gramas.

Segundo Grundy (2006), o menor peso ao nascimento, cerca de 300 gramas no cão de médio porte, está associado ao aumento do risco de mortalidade neonatal, pela imaturidade metabólica do filhote. Possivelmente a hiperlactatemia nos filhotes abaixo do peso é uma indicação de que o sistema aeróbico ainda é imaturo e não está sendo capaz de suprir a demanda de energia requerida, sendo necessária a utilização de mecanismos compensatórios glicólise anaeróbica - (Barroso et al., 2006). O nascimento de neonatos abaixo do peso preconizado para a raça estudada pode ser um indício de problemas de gestação ou fetais de desenvolvimento. É provável que filhotes abaixo do peso tenham dificuldades na adaptação do sistema cardiorrespiratório e na estabilização da ventilação e da perfusão tecidual periférica, o que conduziria à hipóxia e à elevação do lactato.

A concentração lactato, portanto, foi associada à menor pontuação do escore de Apgar (Groppetti et al., 2010), à menor frequência cardíaca fetal (Souza et al., 2006) e, como verificado no presente estudo, ao baixo peso ao nascimento.

Tabela 2. Média e erro-padrão da média (E Pm) do peso (gramas) e da concentração de lactato (mmo/L) obtida pelo método do analisador portátil e da concentração de glicose $(\mathrm{g} / \mathrm{dl})$ de neonatos nascidos de cesariana

\begin{tabular}{lc}
\hline Variável & Média $\pm \mathrm{E} \mathrm{Pm}$ \\
\hline Lactato $(\mathrm{mmol} / \mathrm{L})$ & $9,3 \pm 0,9$ \\
Glicemia $(\mathrm{mg} / \mathrm{dL})$ & $87,8 \pm 6,2$ \\
Peso $(\mathrm{g})$ & $218,5 \pm 12,7$ \\
Glicemia X Lactato & $\mathrm{r}=-0,15 ; \mathrm{P}=0,44$ \\
Peso X Lactato & $\mathrm{r}=-0,67 ; \mathrm{P}=0,0002^{*}$ \\
Peso X Glicemia & $\mathrm{r}=0,04 ; \mathrm{P}=0,82$ \\
\hline$* \mathrm{P} \leq 0,05$ & \multicolumn{2}{|}{}
\end{tabular}

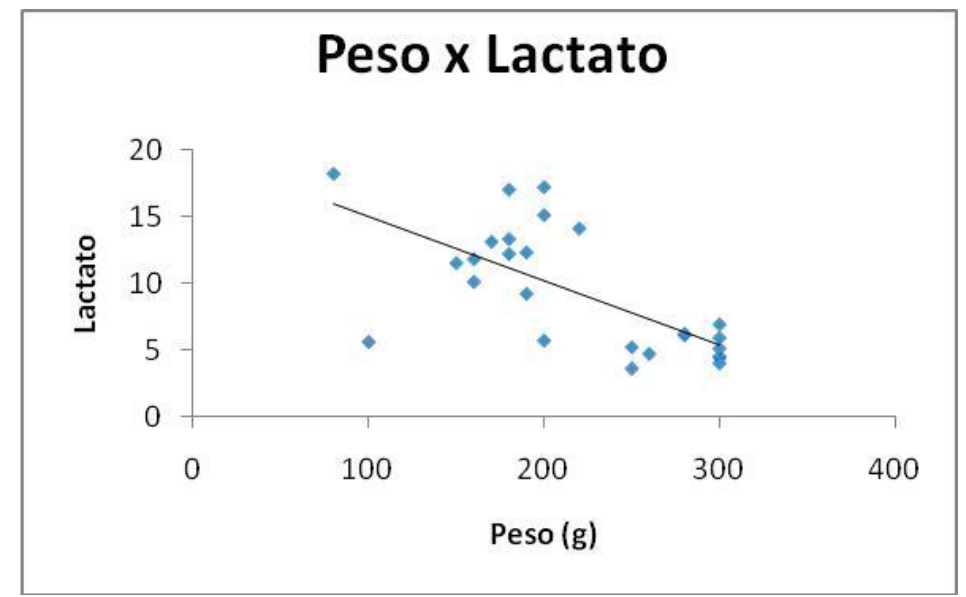

Figura 1. Dispersão da concentração de lactato (mmol/L) associado ao peso dos neonatos

Houve diferença significativa entre os métodos propostos para a análise de lactato. Os resultados verificados com o analisador portátil foram semelhantes aos valores obtidos por Evans (1987), e Hughes et al. (1999) e Hughes (2006), em animais jovens e adultos, respectivamente. Entretanto, os valores de lactado obtidos pelo o analisador portátil foram mais altos quando comparados aos obtidos pelo método enzimático (Tab. 3). Esse resultado apresenta-se de acordo com outro estudo que comparou o lactato obtido pelo medidor portátil e pelo enzimático (RapidLab865) em cães, em que se encontrou uma boa precisão do medidor portátil quando se 
utilizou um volume fixo de sangue de $25 \mu \mathrm{L}$ em vez de uma gota. $\mathrm{O}$ intervalo de referência obtido para o medidor portátil diferiu significativamente em relação aos de referência para o método enzimático, sendo necessário, portanto, o estabelecimento de um padrão para o medidor portátil (Ferasin et al., 2007; Stevenson et al., 2007).

Tabela 3. Média e erro-padrão de médias (E Pm) da concentração de lactado obtidas pelo método enzimático colorimétrico e pelo método de analisador portátil em cadelas submetidas a cesariana

\begin{tabular}{|c|c|c|}
\hline \multirow{2}{*}{ Métodos } & \multicolumn{2}{|c|}{ Lactato $\mathrm{mmo} / \mathrm{L}$} \\
\hline & Média & $\mathrm{P}$ valor \\
\hline Enzimático & $1,6 a \pm 0,6$ & 0.0581 \\
\hline Portátil & $3,5 b \pm 0,6$ & \\
\hline
\end{tabular}

Não houve correlação materna e neonatal entre a concentração de lactato $(\mathrm{r}=0,2 ; \mathrm{P}=0,67)$ e a glicemia $(r=0,48 ; P=0,27)$, sendo o lactato dos neonatos diferente do materno $(\mathrm{P}=0,003)$, semelhante ao descrito por (Souza et al., 2006). O lactato materno não deve ser utilizado como reflexo da hiperlactatemia fetal, pois seus níveis, mesmo dentro da faixa de referência, independentemente do método utilizado, não refletem o estado de perfusão fetal.

\section{CONCLUSÕES}

A mensuração de lactato em neonatos não deve se constituir na única maneira de avaliação da viabilidade neonatal. Sua associação deve ser feita com a história e exame clínico e com o peso ao nascimento. Concentrações elevadas de lactato ao nascimento devem ser interpretadas com cautela, pois podem ser reflexo da acidose respiratória e metabólica que ocorre em quase todos os recém-nascidos, refletindo simplesmente um padrão fisiológico durante a transição fetal-neonatal em cães. A utilização de glicose como medida reanimatória em neonatos caninos nascidos por cesariana deve ser feita sempre mediante a determinação da glicemia, pois os neonatos podem apresentar-se normoglicêmicos.

\section{REFERÊNCIAS}

ALLEN, S.E.; HOLM, J.L. Lactate: physiology and clinical utility. J. Vet. Emerg. Crit. Care, v.18, p.123123,2008

AMERICAN academy of pediatrics. Use and abuse of the Apgar score. Pediatrics, v.78, p.1148-1149, 1986.

BARROSO, R.M.V.; GALLEGO, J.G.; TALHATE, J. et al. A utilização do lactato como marcador biológico prognóstico. UNESC Rev., v.9, p.157-172, 2006.

CASTAGNETTI, C.; PIRRONE, A.; MARIELLA, J. et al. Venous blood lactate evaluation in equine neonatal intensive care. Theriogenology, v.73, p.343357,2010

EVANS, G.O. Plasma lactate measurements in healthy beagle dogs. Am. J. Vet. Res., v.48, p.131-132, 1987.

FERASIN, L.; DODKIN, S.J.; AMODIO, A. et al. Evaluation of a portable lactate analyzer (lactate scout) in dogs. Vet. Clin. Pathol., v.36, p.36-39, 2007.

GORMAN, M.E. Clinical chemistry of the puppy and kitten. In: PETERSON, M.E.; KUTZLER, M.A. (Eds). Small aninal pediatrics: the first 12 months of life. Missouri: Elsevier, 2011. p.259-275.

GROPPETTI, D.; PECILE, A.; DEL CARRO, A.P. et al. Evaluation of newborn canine viability by means of umbilical vein lactate measurement, apgar score and uterine tocodynamometry. Theriogenology, v.74 p.1187-1196, 2010 .

GRUNDY, S.A. Clinically relevant physiology of th neonate. Vet. Clin. N. Am.: Small Anim. Pract., v.36, p.443-459, 2006.

HUGHES, D. Interpretation of Lactate - What is it? What can we do with it? In: NORTH AMERICA VETERINARY CONFERENCE, 2006, Ithaca. Proceedings... Ithaca: NAVC, 2006. North American Veterinary Conference (Eds). (www.tnavc.org). Internet Publisher: International Veterinary Information Service, Ithaca NY (www.ivis.org). Last updated: 11- Jan-2006.

HUGHES, D.; ROZANSKI, E.R.; SHOFER, P.S. Effect of sampling site repeat sampling, $\mathrm{pH}$, and $\mathrm{PCO} 2$ in plasma lactate concentration in healthy dogs. Am. J. Vet. Res., v.60, p.521-524, 1999.

KANEKO, J.J.; HARVEY, J.W.; BRUSS, M.L. Clinical biochemistry of domestic animals. 5.ed. San Diego: Academic, 1997. p.157-203.

KOLISKI, A.; CAT, I.; GIRALDI, D.J. et al. Lactato sérico como marcador prognóstico em crianças gravemente doentes. J. Pediatr., v.81, p.287-292, 2005. 
LAGUTCHIK, M.S.; OGILVIE, G.K.; HACKETT, T.B. et al. Increased lactate concentrations in ill and injured dogs. J. Vet. Emerg. Crit. Care, v.8, p.117$125,1998$.

LAWER, D.F. Neonatal and pediatric care of the puppy and kitten. Theriogenology, v.70, p.384-392, 2008.

LÚCIO, C.F. Influência das condições obstétricas ao nascimento sobre padrões de vitalidade e bioquímica neonatal na espécie canina. 2008. Dissertação (Mestrado em Medicina Veterinária) - São Paulo: Faculdade de Medicina Veterinária e Zootecnia, Universidade de São Paulo, São Paulo.

McMICHAEL, M. Pediatric emergencies. Vet. Clin. N. Am.: Small Anim. Pract., v.35, p.421-434, 2005.

McMICHAEL, M.A.; LEES, G.E.; HENNESSEY, J. et al. Serial plasma lactate concentrations in 68 puppies aged 4 to 80 days. J. Vet. Emerg. Crit. Care, v.15, p.17-21, 2005.
PANG, D.S.; BOYSEN, S. Lactate in veterinary critical care: pathophysiology and management. J. Am. Anim. Hosp. Assoc., v.43, p.270-279, 2007.

SOUZA, P.S.; OLIVA, V.N.L.S.; FERREIRA, J.Z. et al. Correlação do lactato sérico com a vitalidade de neonatos de cães nascidos de cesariana sob anestesia geral inalatória. In: CONGRESSO DE INICIAÇÃO CIENTÍFICA DA UNESP, 22., 2006. Disponível em: <http://www.prope.unesp.br/xxii_cic/ver_resumo.php? area $=100043 \&$ subarea...

STEVENSON, C.K.; KIDNEY, B.A.; DUKE, T. et al. Evaluation of accutrend for lactate mensurement in dogs. Vet. Clin. Pathol., v.36, p.261-266, 2007.

VIVAN, M.C.R.; VIDES, J.P.; SOUZA, T.F.B. et al. Umbilical cord blood lactate in the assessment of fetal stress during delivery in dogs. In: WORLD CONGRESS OF VETERINARY ANAESTHESIOLOGY, 2009, Glasgow. Proceedings... Glasgow, 2009. 